\title{
DÉPOSITION PAR PULVÉRISATION CATHODIQUE RADIO FRÉQUENCE ET CARACTÉRISATION ÉLECTRONIQUE, STRUCTURALE ET OPTIQUE DE COUCHES MINCES DU DIOXYDE DE TITANE
}

\author{
K. HAFIDI*, M. AZIZAN, Y. IJDIYAOU and E. L. AMEZIANE \\ Laboratoire de Physique du Solide et des Couches Minces, Faculté des Sciences Semlalia, \\ Université Cadi Ayyad, BP 2390 Marrakech, Maroc
}

(Received 8 May 2003)

\begin{abstract}
Deposited titanium oxide thin films are used as optical protector films for several materials and as energy converters for solar cells. In this work, titanium oxide thin films are deposited on c-Si and glass substrates by reactive radiofrequency sputtering. All the deposits are grown at ambient temperature and the sputtering gas is a mixture of oxygen and argon with an overall pressure of $10^{-2} \mathrm{mbar}$. The oxygen partial pressure ratios varies from $5 \%$ to $20 \%$.

Characterization of deposited films is made by grazing incidence X-ray diffraction (GIXD), grazing incidence X-ray reflection (GIXR), X-ray photoemission spectroscopy (XPS) and optical transmission spectroscopy. The characterization results reveal that deposited films of $\mathrm{TiO}_{2}$ are polycrystalline and present both rutile and anatase phases. The chemical composition of raw films in Ti:O ratio is equal to 1:2.02, and the titanium at surface is completely oxidized. In fact, the Ti2p core level behavior shows that the oxidization state of $\mathrm{Ti}$ is equal to +4 .

The specularily reflected intensity according to incidence angle of the X-ray on $\mathrm{TiO}_{2} /$ glass structure shows one critical angle attributed to the $\mathrm{TiO}_{2}$ film equal to $0.283^{\circ}$. This angle value involves film density between rutile and anatase phases. The optical characterization shows that $\mathrm{TiO}_{2}$ thin films obtained are transparent in visible range, and have a refraction index value equal to 2.45 and when extrapolated to infrared range, it is equal to 2.23 . The value of gap energy $(3.35 \mathrm{eV})$ is deduced from variation of absorption coefficient versus incident radiation energy.
\end{abstract}

Keywords: Titanium oxide $\left(\mathrm{TiO}_{2}\right)$; Thin film; Sputtering; Solar cells; Spectroscopy

Dans cet article, nous présentons une étude de caractérisation des couches minces d'oxyde de titane dans les structures $\mathrm{TiO}_{2} / \mathrm{c}-\mathrm{Si}$ et $\mathrm{TiO}_{2} /$ verre, déposées par pulvérisation cathodique radio fréquence réactive.

Les spectres de diffraction des rayons $\mathrm{X}$ en incidence rasante montrent que nos couches sont constituées d'un mélange polycristallin de deux phases du dioxyde de titane: la rutile et l'anatase. Les mesures de réflectométrie des rayons X mettent en évidence un seul angle limite de réflexion totale égal à $0.283^{\circ}$. Cet angle limite correspond à une densité massique comprise entre celle de la phase rutile et celle de la phase anatase.

Les analyses XPS des films à l'état brut indiquent que la composition chimique Ti:O est de 1:2.02. Le niveau de cœur Ti2p montre que l'état d'oxydation du Ti est +4 .

Les films $\mathrm{TiO}_{2}$ obtenus sont transparents dans le visible et sont caractérisés par un indice de réfraction extrapolé à l'infini (infrarouge) de 2.23, un indice de réfraction dans le visible de 2.45 et une bande interdite de largeur $3.35 \mathrm{eV}$.

Mots clés: $\mathrm{TiO}_{2}$, couche mince, pulvérisation cathodique, cellule photovoltaïque, spectroscopie

* Corresponding author. E-mail: k.hafidi@ucam.ac.ma 


\section{INTRODUCTION}

Le dioxyde de titane $\left(\mathrm{TiO}_{2}\right)$ est un matériau très sollicité pour ses propriétés optiques, mécaniques et électroniques. On le retrouve très souvent dans des domaines pratiques telles que les couches antireflets, la conversion d'énergie solaire, les guides d'onde, et les électrodes (grille) dans les dispositifs MOS. En effet, il présente des propriétés physico-chimiques et électriques intéressantes [1-4]: il présente une bonne adhérence, une excellente dureté mécanique, une bonne stabilité chimique, un fort indice de réfraction et une bonne transparence dans le domaine du visible et du proche infrarouge. Ce matériau a fait l'objet de nombreux travaux de recherches [5-10]. Ses propriétés cristallographiques, électroniques et optiques ont été explorées par différentes techniques d'analyses. De ses propriétés cristallines, nous rappelons que le $\mathrm{TiO}_{2}$ en couche mince cristallise sous trois formes: la rutile (tetragonal), l'anatase (tetragonal) ou la brookite (orthorhombique). La phase rutile est reconnue comme étant la plus stable alors que la phase anatase est la moins stable.

La plus part des travaux antérieures portent essentiellement sur les films de $\mathrm{TiO}_{2}$ cristallisés dans la phases anatase ou dans la phase rutile. La cristallisation dans l'une des deux phase nécessite des conditions très particulières de la pression partielle d'oxygène, de la température et de la vitesse de dépôt ainsi que de la température de recuit.

La principale motivation du présent travail est l'exploration des propriétés électroniques, structurales et optiques des dépôts en couches minces de $\mathrm{TiO}_{2}$ en présence des deux phases anatase et rutile. Ces couches sont élaborées par la méthode de pulvérisation cathodique radio fréquence réactive à différentes pressions partielles d'oxygène. La caractérisation est effectuée sur des échantillons à l'état brut sans aucun traitement thermique préalable.

Pour la première fois, la réflectométrie et la diffraction des rayons $\mathrm{X}$ en incidence très rasante ont été utilisées simultanément pour la caractérisation des couches minces de $\mathrm{TiO}_{2}$. Ainsi, la diffraction des rayons $\mathrm{X}$ en incidence très rasante nous a permis de dégager le profil des phases rutile et anatase en faisant varier l'angle d'incidence $\alpha$ de $0.15^{\circ}$ à $0.8^{\circ}$ (autrement dit en faisant varier la profondeur de pénétration des rayons $\mathrm{X}$ de $30 \AA$ à $1000 \AA$ ). La réflectométrie des rayons $\mathrm{X}$, quant à elle, nous a permis d'atteindre d'une façon précise la densité et l'épaisseur de nos couches.

\section{PROCÉDURE EXPÉRIMENTALE}

Les films minces d'oxyde de titane $\left(\mathrm{TiO}_{2}\right)$, dans les structures $\mathrm{TiO}_{2} / \mathrm{c}-\mathrm{Si}$ et $\mathrm{TiO}_{2} /$ verre, sont déposés, à partir d'une cible de titane de haute pureté $(5 \mathrm{~N})$, dans une enceinte de pulvérisation cathodique radio fréquence. L'enceinte est mise sous un vide secondaire de $10^{-7} \mathrm{mbar}$ avant l'introduction d'un plasma formé d'un mélange d'argon $(5 \mathrm{~N})$ et d'oxygène $(5 \mathrm{~N})$ à une pression totale de $10^{-2}$ mbar. Les essais ont été effectués à la température ambiante et à des proportions d'oxygène dans le mélange de 5\%,10\% et $20 \%$.

La caractérisation de nos échantillons a été achevée à l'aide des techniques d'analyses complémentaires suivantes: la diffraction des rayons $X$ en incidence très rasante (GIXD), la réflectométrie des rayons $\mathrm{X}$ en incidence très rasante (GIXR), la spectroscopie de photoémission X (XPS), et la spectroscopie de transmission optique.

La diffraction des rayons $X$ en incidence très rasante est une technique non destructive et très puissante pour la détermination de la structure cristallographique des composés formés à la surface et à l'interface. Elle donne également la structure cristallographique du volume. Ceci est obtenu en faisant varier l'angle d'incidence des rayons $\mathrm{X}$ de $0.15^{\circ}$ à $0.8^{\circ}$, ce qui permet de faire varier la profondeur de pénétration des rayons $\mathrm{X}$ de quelques dizaines 
d'Angström jusqu'à quelques centaines d'Angström. Nous avons utilisé cette technique d'analyse pour déterminer la structure cristallographique de la surface et du volume des couches d'oxyde de titane produites à différentes pressions partielles d'oxygène. Alors que la réflectométrie des rayons $X$ en incidence très rasante permet d'évaluer d'une façon précise la densité et l'épaisseur de nos couches. Cette technique non destructive consiste à envoyer, sous une incidence très rasante, un faisceau de rayons $X$ parallèles et filtrés sur l'échantillon puis à enregistrer les variations d'intensité du faisceau réfléchi spéculairement en fonction de $\alpha$.

Dans les expériences de réflectométrie et de diffraction de rayons $\mathrm{X}$ en incidence très rasante, nous avons utilisé un générateur de rayons $\mathrm{X}$ à anode de cuivre équipé d'un foyer linéaire de $0.1 \times 10 \mathrm{~mm}^{2}$ et un filtre en Nickel qui nous permet de sélectionner la raie d'émission $\mathrm{Cu} \mathrm{K} \mathrm{K}_{\alpha}$. Le faisceau de rayons $\mathrm{X}$ est collimaté par un système de fente fine en tantale de largeur variable. Dans le cas de la réflectométrie, cette largeur est comprise entre 10 et 40 $\mu \mathrm{m}$, alors qu'elle varie de 100 à $300 \mu \mathrm{m}$ dans le cas de la diffraction. Le dispositif est muni d'un goniomètre $\theta-2 \theta$ à bras découplés, d'un détecteur à scintillation et d'un détecteur solide $\mathrm{Si}$-Li. Le pilotage du goniomètre et l'acquisition des donnés sont assurés par un micro-ordinateur.

Dans l'exploitation des résultats nous avons pris en considération la divergence angulaire qui résulte de l'utilisation des fentes de tantale et de la précision angulaire des deux rotations du goniomètre (relatifs au détecteur et à l'échantillon). Les valeurs de ces grandeurs ont été déterminées dans nos travaux antérieurs $[11,12]$. Elles sont de $0.02^{\circ}$ pour la divergence angulaire et de $0.003^{\circ}$ pour la précision angulaire du goniomètre.

La structure électronique et la composition chimique des couches en atomes de titane (Ti) et d'oxygène $(\mathrm{O})$ dans les films $\mathrm{TiO}_{2}$ ont été déterminées à l'aide de la spectroscopie de photoémission $\mathrm{X}$. Cette technique consiste à exciter par un rayonnement monochromatique les électrons d'un solide et à recueillir ceux qui peuvent franchir la barrière de potentiel de surface.

Les constantes optiques des couches minces de $\mathrm{TiO}_{2}$ ont été étudiées à l'aide de la spectroscopie de transmission optique, une technique connue comme étant simple et non destructive. Elle consiste à mesurer le spectre transmis par l'échantillon à l'aide d'un spectrophotomètre relié à une centrale d'acquisition des données. Le spectrophotomètre utilisé dans nos expériences est de type Shimadzu UV-3101PC. Il fonctionne à double faisceau et couvre la gamme spectrale $190-3200 \mathrm{~nm}$.

\section{RÉSULTATS EXPÉRIMENTAUX ET DISCUSSIONS}

\section{Etude Par la Diffraction Des Rayons X en Incidence Rasante}

Nous avons analysé des structures de type $\mathrm{TiO}_{2} / \mathrm{c}-\mathrm{Si}$ et $\mathrm{TiO}_{2} /$ verre obtenues à des pressions partielles d'oxygène de $2 \cdot 10^{-3}$ mbar, $10^{-3}$ mbar et $5 \cdot 10^{-4}$ mbar.

Les spectres de diffraction des rayons $\mathrm{X}$ en incidence très rasante obtenus, à différents angles d'incidences, sur les échantillons $\mathrm{TiO}_{2} / \mathrm{c}$-Si non traités thermiquement sont représentés sur la Figure 1. Les Figures 1a et $1 \mathrm{~b}$, relatives respectivement aux pressions partielles d'oxygène $10^{-3}$ et $2 \cdot 10^{-3}$, mettent en évidence la présence des deux phases de $\mathrm{TiO}_{2}$ : l'anatase indiquée par la présence des raies (101), (103), (004), (200), (105), (211) et (213), et la rutile indiquée par la présence des raies (110), (211), (220), (301) et (112). L'évolution des spectres de diffraction en fonction de l'angle d'incidence rasante met en évidence une variation continue des proportions des deux phases lorsqu'on se déplace de la surface vers l'intérieur des échantillons. Le mélange des deux phases rutile et anatase avait été également obtenu par Schiller \& al [13] dans des 


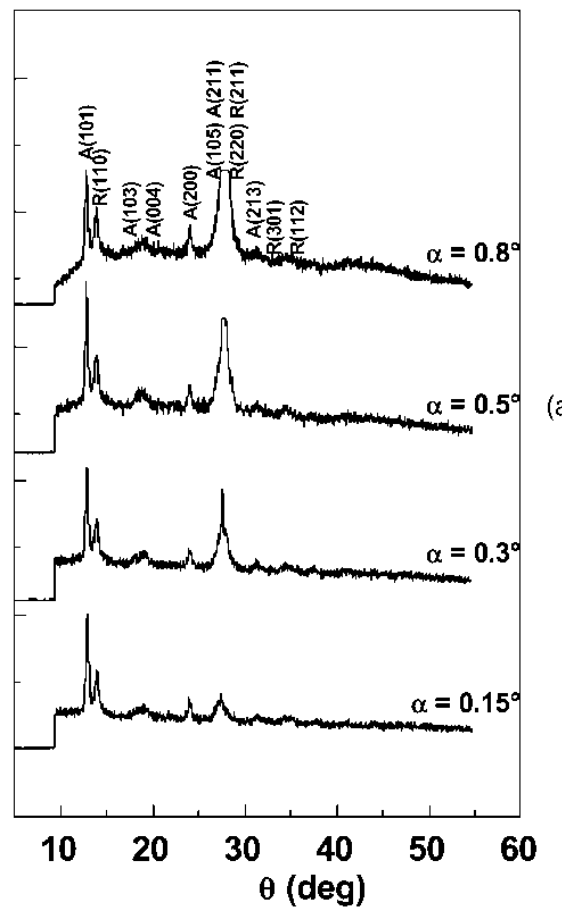

(a)

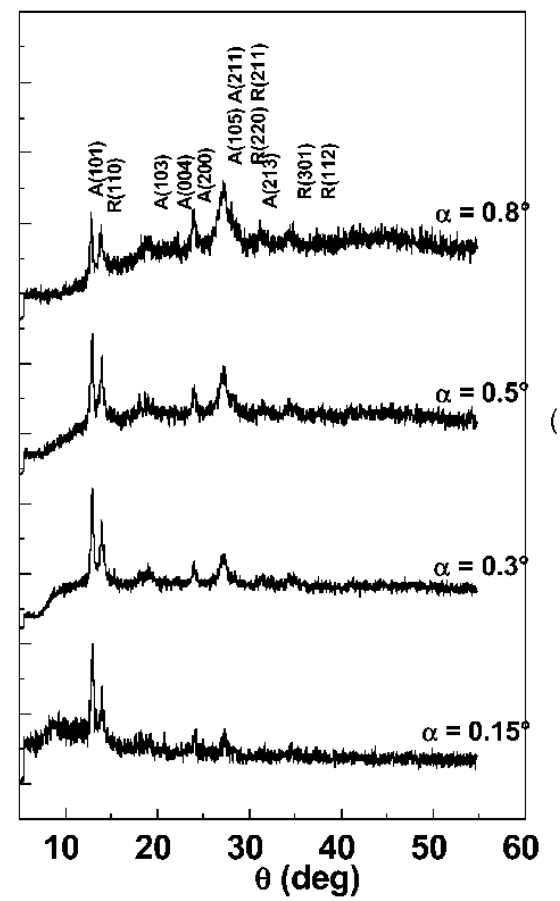

FIGURE 1 Spectres de diffraction des rayons $X$ en incidence rasante $\left(\alpha=0.15 ; 0.3 ; 0.5 ; 0.8^{\circ}\right)$ des structures $\mathrm{TiO}_{2} / \mathrm{c}-\mathrm{Si}$ obtenues aux pressions partielles d'oxygène suivantes: (a) $10^{-3} \mathrm{mbar}$, (b) $2 \cdot 10^{-3} \mathrm{mbar}$ et (c) $5 \cdot 10^{-4}$ mbar. 


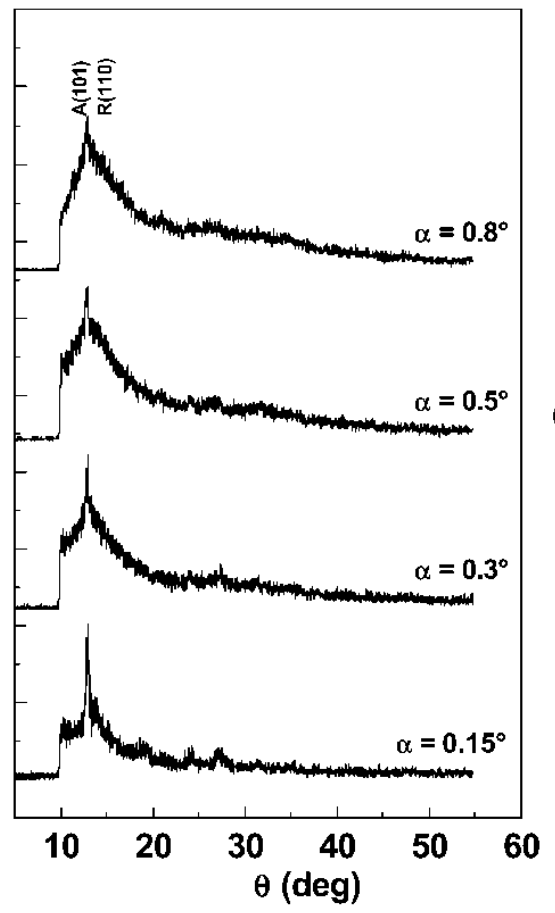

(c)

FIGURE 1 (Continued).

couches d'oxydes de titane élaborées par la méthode "reactive dc plasmatron sputtering" à de faibles pressions partielles d'oxygène et à des températures du substrat comprises entre $25^{\circ} \mathrm{C}-500^{\circ} \mathrm{C}$.

En effet, si on compare les intensités des raies (101) de la phase anatase et (110) de la phase rutile aux angles d'incidences $\alpha=0.15^{\circ}, 0.3^{\circ}, 0.5^{\circ}$ et $0.8^{\circ}$ on constate une augmentation de l'intensité de la raie (110) caractéristique de la phase rutile au dépend de la raie (101) relative à la phase anatase. Autrement dit, la croissance de la phase rutile devient de plus en plus importante au fur et à mesure que l'on pénètre à l'intérieur de l'échantillon. Ceci peut être justifié par le faite que le substrat, et sous l'effet du bombardement ionique lors du dépôt, peut atteindre une température de $100^{\circ} \mathrm{C}$. Ainsi les premières monocouches déposées subissent un recuit thermique durant un temps plus important que celui des monocouches surfaciques déposées en dernier lieu avant l'interruption du dépôt. Le recuit étant connu comme un facteur qui favorise la formation de la phase rutile $[14,15]$.

La Figure 1c présente les spectres de diffraction mesurés sur une couche d'oxyde de titane déposée sur un substrat de silicium sous une pression partielle d'oxygène de $5 \cdot 10^{-4}$ mbar. Afin d'obtenir la structure cristallographique à la surface, la diffraction a été effectuée à un angle d'incidence $\alpha=0.15^{\circ}$. Le spectre obtenu est dominé par la raie fine caractéristique de la phase anatase, ce qui prouve que la surface est essentiellement composée de la phase anatase de $\mathrm{TiO}_{2}$. Lorsque l'angle d'incidence $\alpha$ augmente, on note l'émergence d'une raie large, attribuée à la structure rutile, dont le barycentre se situe à la position de la raie (110). Quand l'angle d'incidence atteint la valeur $\alpha=0.8^{\circ}$ cette structure domine entièrement le spectre de diffraction. La valeur importante de la largeur de cette raie dans les spectres obtenus montre que le volume des couches élaborées est essentiellement constitué de la phase rutile mal cristallisée. La relation de Deby-Scherrer [16] permet d'évaluer la taille des cristallites de la rutile à $30 \AA ̊$. La structure désordonnée de la rutile est à corréler avec la faible quantité 
d'oxygène dans le plasma et qui n'est pas suffisante pour oxyder tous les atomes de titane. A cette même pression d'oxygène $\left(\mathrm{PO}_{2}=5 \cdot 10^{-4} \mathrm{mbar}\right)$, et lorsque le dépôt est effectué sur un substrat de verre, les spectres obtenus sont identiques à ceux des couches déposées sur un substrat de silicium à des pressions d'oxygène plus élevées $\left(10^{-3}\right.$ et $2 \cdot 10^{-3}$ mbar) (Figs. (1a) et (1b)). Le substrat en verre constitue un réservoir d'oxygène et permet de combler le déficit en oxygène en provenance du plasma, ce qui explique l'oxydation totale du titane.

Létude expérimentale qui suit a été menée uniquement sur des échantillons élaborés sous $10^{-3}$ mbar de pression partielle d'oxygène.

\section{Etude Par la Réflectrométrie Des Rayons X}

Sur la Figure 2a, nous avons représenté la courbe de variation de l'intensité des rayons $\mathrm{X}$ réfléchis spéculairement par la structure $\mathrm{TiO}_{2} / \mathrm{c}-\mathrm{Si}$ en fonction de l'angle d'incidence rasante $\alpha$. Cette courbe fait apparaître un seul palier de réflexion totale, ce qui confirme le caractère homogène de nos films. L'angle limite de réflexion totale correspondant est égal à $0.283^{\circ}$. La mesure de l'angle critique de réflexion totale permet de déterminer la densité électronique de nos couches et par conséquent leur densité massique [17]. Ainsi, et d'après les résultats obtenus, la densité massique de nos couches est de $3.92 \mathrm{~g} / \mathrm{cm}^{3}$. Cette valeur est comprise entre celles des densités massiques des deux phases cristallines du $\mathrm{TiO}_{2}$ [18]. En plus, le pourcentage moyen de la phase anatase dans le mélange est de $72 \%$. Ces résultats sont en bon accord avec les mesures de diffraction des rayons $\mathrm{X}$ en incidence rasante (Fig. 1).

La Figure $2 b$ représente la courbe d'évolution semi-logarithmique de l'intensité réfléchie par la structure $\mathrm{TiO}_{2} / \mathrm{c}$-Si en fonction de l'angle d'incidence $\alpha$ supérieur à $0.35^{\circ}$. Sur cette courbe, on observe une succession de maxima et de minima dite franges d'interférences de Kiessing. Ces franges sont dus au phénomène d'interférence entre les ondes réfléchies par les dioptres air/ $\mathrm{TiO}_{2}$ et $\mathrm{TiO}_{2} / \mathrm{c}$-Si. Notons que l'épaisseur de la couche déposée peut être déterminée à partir des positions angulaires des franges de Kiessing. Une estimation de l'épaisseur e a été déterminée en utilisant la relation [17, 19]:

$$
e=\frac{\lambda}{2 \Delta \theta}
$$

Avec: $\Delta \theta$ est l'interfrange angulaire; $\lambda$ est la longueur d'onde de la radiation $\mathrm{K}_{\alpha} \mathrm{du}$ cuivre.

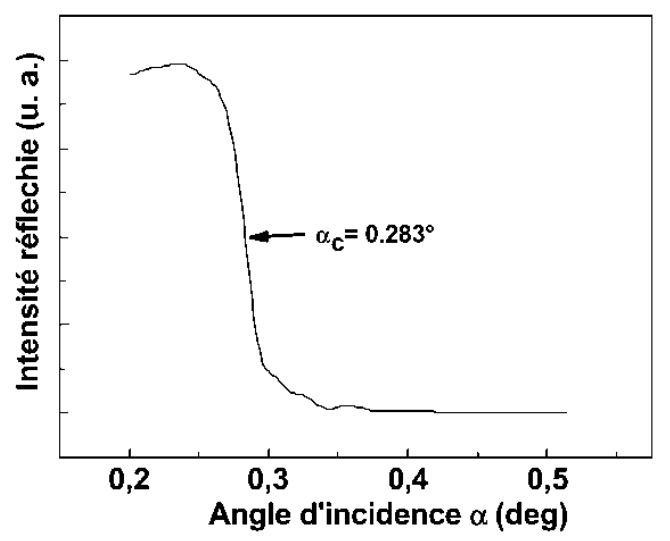

FIGURE 2a Variation linéaire de l'intensité réfléchie spéculairement en fonction de l'angle d'incidence $\alpha$ de l'échantillon $\mathrm{TiO}_{2} / \mathrm{c}-\mathrm{Si}$. 


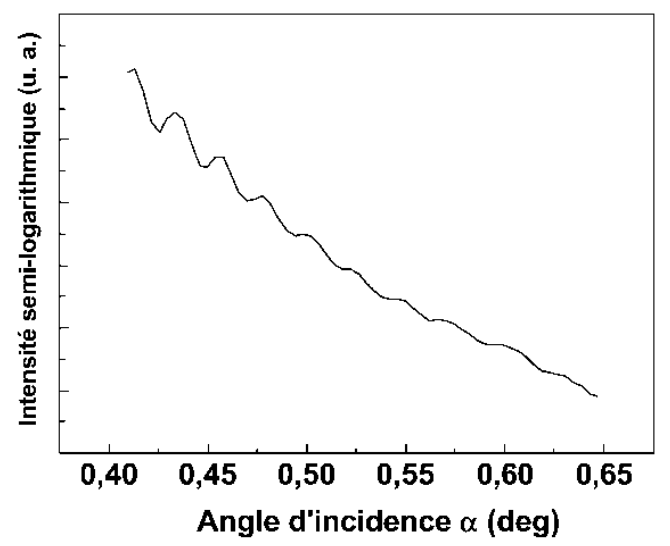

FIGURE $2 \mathrm{~b}$ Variation semi-logarithmique de l'intensité réfléchie spéculairement en fonction de l'angle d'incidence $\alpha$ de l'échantillon $\mathrm{TiO}_{2} / \mathrm{c}-\mathrm{Si}$.

Ainsi, l'épaisseur calculée de notre couche est de 2050 Å. Cette valeur sera considérée par la suite dans l'exploitation des mesures optique.

\section{Etude Par la Spectroscopie de Photoémission X}

Nous avons déterminé la composition chimique de nos couches $\mathrm{TiO}_{2} / \mathrm{c}-\mathrm{Si}$ brut en comparant les intensités des deux niveaux de cœur Ti2 $\mathrm{p}\left(\mathrm{I}_{\mathrm{Ti}}\right)$ et $\mathrm{O} 1 \mathrm{~s}\left(\mathrm{I}_{\mathrm{O}}\right)$. Le rapport des concentrations relatives des deux éléments qui constituent nos films (le titane et l'oxygène) est donné par la relation:

$$
\frac{N_{\mathrm{O}}}{N_{\mathrm{Ti}}}=\frac{I_{\mathrm{O}}}{I_{\mathrm{Ti}}} \frac{\sigma_{\mathrm{Ti}}}{\sigma_{\mathrm{O}}} \frac{\lambda_{\mathrm{Ti}}}{\lambda_{\mathrm{O}}} \frac{T_{\mathrm{Ti}}}{T_{\mathrm{O}}}
$$

où: $N_{\mathrm{O}}$ et $N_{\mathrm{Ti}}$ sont respectivement les densités atomiques en oxygène et en titane; $\sigma_{\mathrm{O}}$ et $\sigma_{\mathrm{Ti}}$ représentent respectivement les sections efficaces d'ionisation des niveaux de cœur O1s et Ti2 $\mathrm{p}_{3 / 2}$. Dans le cas d'une raie d'excitation $\mathrm{MgK}_{\alpha}(1253.6 \mathrm{eV})$, ces deux grandeurs valent respectivement 2.85 et 5.22 [20]; $T$ est le facteur de transmission qui est inversement proportionnelle à la racine carrée de l'énergie cinétique du niveau de cœur correspondant [21]; $\lambda$ étant le libre parcours moyen inélastique du photoélectron. Il est fonction de l'énergie cinétique et de la nature du milieu de création du photoélectron. Puisque les photoélectrons O1s et Ti2p sont issus du même matériau avec des énergies cinétiques très voisines, leurs libres parcours moyen inélastique ont pratiquement la même valeur.

Le rapport de concentration atomique $N_{\mathrm{O}} / N_{\mathrm{T}}$ est calculé à partir du rapport des intensités $\mathrm{I}_{\mathrm{O}} / \mathrm{I}_{\mathrm{Ti}}$ (Fig. 3a). Dans notre cas, ce rapport atteint la valeur de 2,02 caractéristique des couches de $\mathrm{TiO}_{2}$ stœchiométriques.

A la surface de l'échantillon brut, le niveau de cœur Ti2p, représenté par la Figure 3b, révèle la présence de la double structure Ti2 $\mathrm{p}_{3 / 2}$ - Ti2 $\mathrm{p}_{1 / 2}$ due au couplage spin orbite. Ces deux pics sont centrés aux énergies de liaisons $458.4 \mathrm{eV}$ et $464 \mathrm{eV}$. Le déplacement chimique du niveau de cœur $T i 2 \mathrm{p}_{3 / 2}$ ainsi que la différence des énergies de liaison $\left(\mathrm{E}_{1}\right.$ Ti2p3/2- $\left.\mathrm{E}_{1} \mathrm{Ti2p} 1 / 2\right)$ montrent que le titane est dans l'état d'oxydation caractéristique de $\mathrm{Ti}^{4+}$ dans le $\mathrm{TiO}_{2}[22]$. C'est à dire que le titane est entièrement oxydé. 


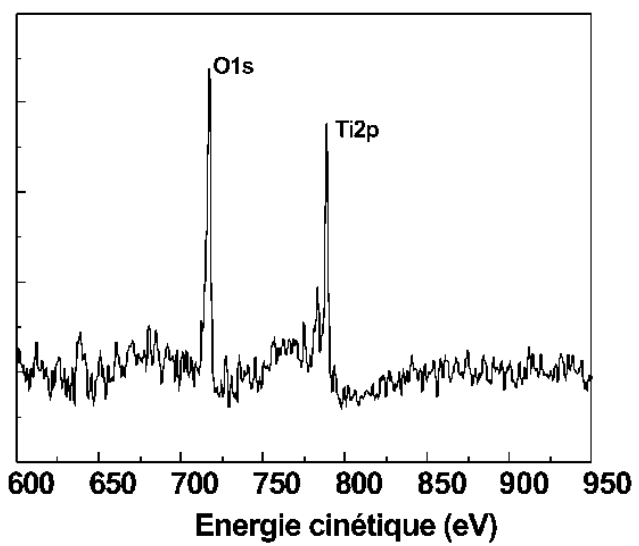

FIGURE 3a Spectre expérimental d'XPS des niveaux de cœur Ti2p et O1s de la structure $\mathrm{TiO}_{2} / \mathrm{c}-\mathrm{Si}$.

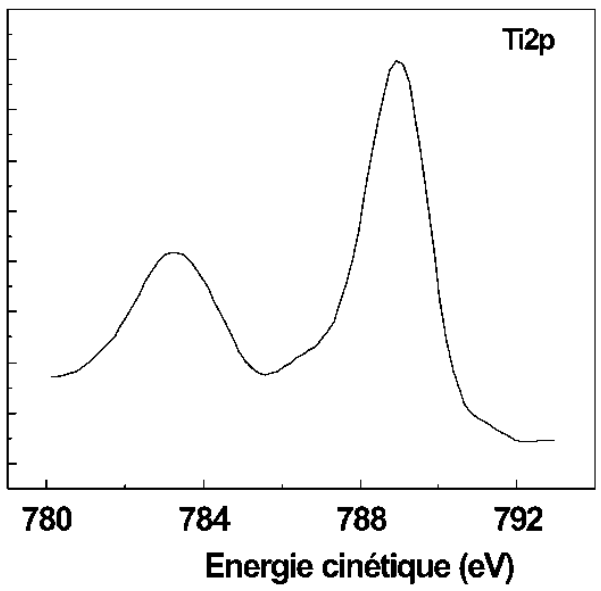

FIGURE 3b Spectre du niveau de cœur Ti2p dans le $\mathrm{TiO}_{2}$.

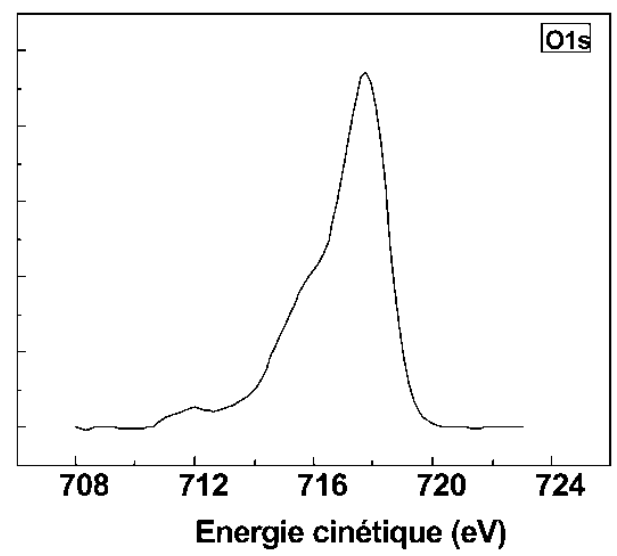

FIGURE 3c Spectre du niveau de cœur O1s dans le $\mathrm{TiO}_{2}$ 
Le spectre du niveau de cœur $\mathrm{O} 1 \mathrm{~s}$ enregistré pour l'échantillon brut est légèrement asymétrique (voir Figure 3c). Il présente un épaulement de faible intensité du coté des hautes énergies de liaisons. Cet épaulement est centré à $531.5 \mathrm{eV}$. Comme c'est reporté en littérature [23], il peut être dû aux groupements hydroxydes et/ou à l'oxygène chimisorbé.

\section{Etude Par la Spectroscopie de Transmission Optique}

Le dioxyde de titane est largement utilisé comme couche protectrice et/ou antireflet [24,25]. Le paramètre important dans ces applications est l'indice de réfraction. Plus l'indice de réfraction est élevé plus les performances optique du matériau sont meilleurs.

La Figure 4a montre le spectre de transmission typique des couches minces de l'oxyde de titane $\mathrm{TiO}_{2}$, obtenues sous une pression partielle d'oxygène de $10^{-3}$ mbar, déposées sur des substrats de verre Corning. Ce spectre indique que les films minces de $\mathrm{TiO}_{2}$ sont transparents dans le visible.

Les oscillations observées sur le spectre de transmission $T(\lambda)$ sont dues aux phénomènes d'interférences et dépendent de l'indice de réfraction de la couche étudiée. L'analyse de ce genre de spectres permet de déterminer l'épaisseur (e), l'indice (n), le coefficient d'extinction (k) et le coefficient d'absorption $(\alpha)$ de la couche.

Les constantes optiques sont calculées en utilisant une seule grandeur spectrophotométrique: la transmission optique. La méthode de calcul que nous avons utilisé a été développée au sein de notre laboratoire [26]. Elle ne tient pas compte simultanément des modèles de rugosité et des inhomogéneités d'épaisseur et d'indice de réfraction. Dans ce travail, cette méthode est utilisée dans le cas d'une variation continue d'épaisseur dans les films. En effet, les échantillons élaborés par pulvérisation cathodique radio fréquence sont généralement lisses par contre, ils présentent des inhomogéniétés d'épaisseur.

$\mathrm{Si}$ on néglige l'erreur due à la détermination des positions des franges d'interférences, nous pouvons dire que l'incertitude sur l'indice de réfraction est essentiellement due à l'incertitude sur l'épaisseur. De plus, l'erreur sur l'épaisseur est d'autant plus importante que le nombre des franges est minime. Ainsi, il est intéressant de prévoir une méthode de mesure précise de l'épaisseur telle que la réflectométrie des rayons $\mathrm{X}$ en incidence rasante pour pouvoir augmenter la certitude de la valeur de l'indice de réfraction.

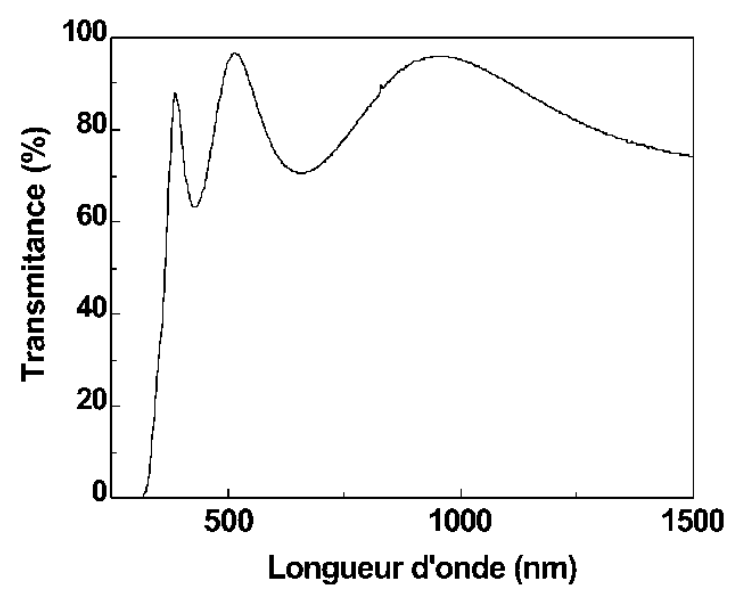

FIGURE 4a Spectre de transmission optique de l'échantillon $\mathrm{TiO}_{2} /$ Corning. 


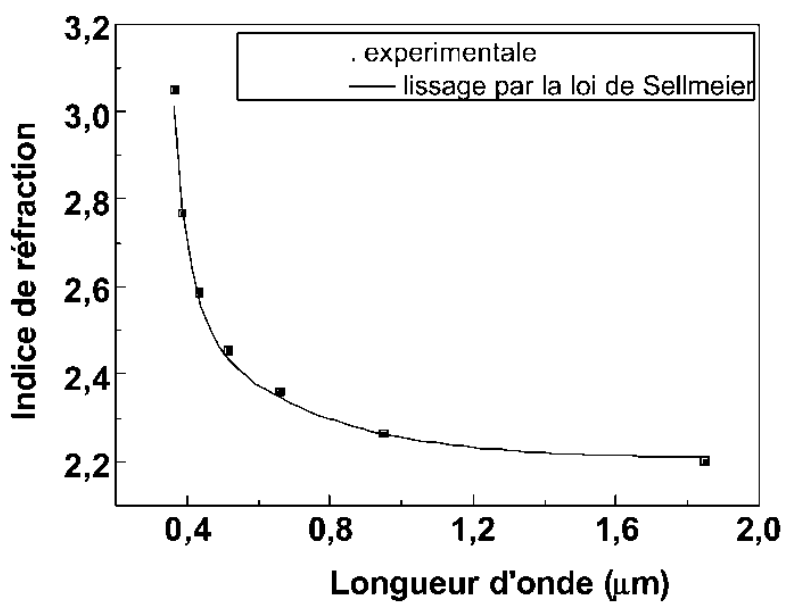

FIGURE $4 \mathrm{~b}$ Indice de réfraction calculé sur chaque frange d'interférence et lissage des points expérimentaux par la loi de Sellmeier.

L'indice de réfraction, calculé pour chaque frange d'interférence du spectre de transmission, est représenté sur la Figure $4 \mathrm{~b}$. Sur la même figure nous avons représenté l'indice théorique donné par la loi de dispersion de Sellmeier [27]:

$$
n^{2}(\lambda)=n_{\infty}^{2}+\frac{b^{2}}{\lambda^{2}-\lambda_{0}^{2}}
$$

Où $n_{\propto}$ est l'indice extrapolé à l'infini, b et $\lambda_{0}$ sont des constantes.

Les valeurs de l'indice $n_{\propto}$ et la variation continue d'épaisseur $\sigma$, sont reportées sur le tableau I.

Pour déterminer l'effet de la méthode d'élaboration sur les propriétés optiques des films minces de $\mathrm{TiO}_{2}$, Bennet \& al. [8] ont utilisé différentes techniques. Ils ont montré que l'indice de réfraction dépend étroitement de la technique de dépôt. Ainsi, les couches minces obtenues par évaporation par jet d'électrons (electron beam evaporation) ont un indice de réfraction faible $(n=2,18)$ alors que l'indice le plus élevé est obtenu pour les films préparés par pulvérisation $(n=2,46)$ et par implantation ionique $\ll$ ion planted $\gg(n=2,61)$. Ces résultats ont été, par la suite, confirmés par Laube \& al. [28].

D'autres facteurs opératoires, telle que la température, influent sur l'indice de réfraction. En effet, différents travaux ont montré que l'indice $\mathrm{n}$ augmente avec la température du recuit [14] et avec la température du substrat lors du dépôt [14,29].

La Figure 4c montre la courbe de variation du coefficient d'absorption $\alpha$ en fonction de l'énergie des photons $h v$. Cette courbe nous a permis par la suite de déterminer la nature et la valeur du gap optique de nos couches.

TABLEAU I Constantes Optiques de nos Couches Minces de $\mathrm{TiO}_{2}$ Obtenues par la Méthode des Interférences Optiques.

\begin{tabular}{ll}
\hline$n$ r & 2.23 \\
$\sigma(\AA)$ & 82 \\
$\lambda_{0}(\mathrm{~m} \mu)$ & 0.3154 \\
$b(\mu \mathrm{m})$ & 0.3846
\end{tabular}




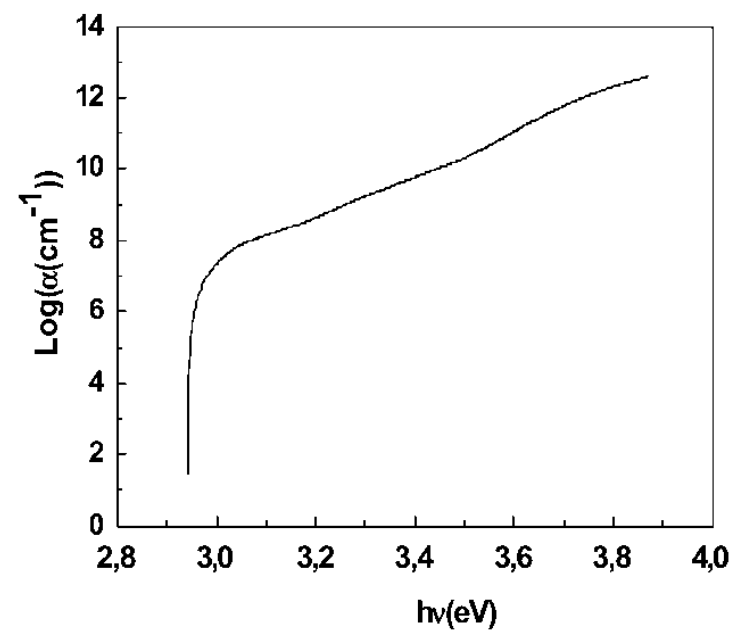

FIGURE 4c Coefficient d'absorption en fonction de l'énergie $\mathrm{h} v$ de l'échantillon $\mathrm{TiO}_{2} /$ Corning.

Le modèle de Tauc qui caractérise les transitions optiques indirectes: $\left((\alpha(h v))^{0.5}=f(h v)\right.$; où $\alpha$ est le coefficient d'absorption) permet de simuler d'une façon précise les variations du coefficients d'absorption dans le cas de nos films de $\mathrm{TiO}_{2}$. Ce constat a été également établi par Tang \& al. [30] qui confirment que le coefficient d'absorption des couches minces de $\mathrm{TiO}_{2}$ élaborées par pulvérisation varient en $(h v)^{2}$.

Pour déterminer la valeur de la bande interdite, nous avons représenté les résultats de la figure $4 \mathrm{c}$ sous la forme $(\alpha(h v))^{0.5}$ en fonction de $\mathrm{h} v$ pour des valeurs de $\alpha$ supérieur à $10^{4} \mathrm{~cm}^{-1}$ (Figure $4 \mathrm{~d}$ ). Ainsi, la largeur de la bande interdite est déterminée par extrapolation de la droite $(\alpha(h v))^{0.5}=f(h v)$ à la valeur $\alpha=0$. Par cette méthode, les couches déposées sont caractérisées par une bande interdite de $3.35 \mathrm{eV}$.

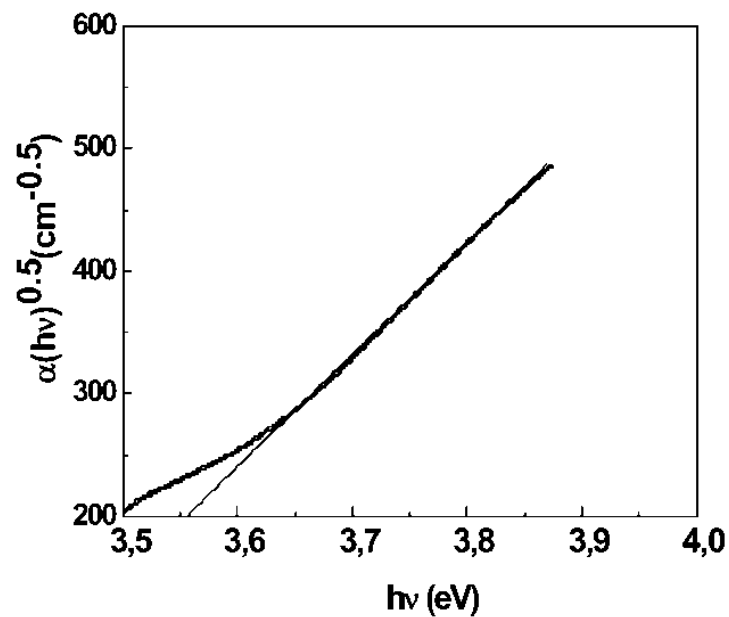

FIGURE 4d Représentation des données de la Figure 4c sous la forme $(\alpha(h v))^{0.5}=f(h v)$. 


\section{CONCLUSION}

Les couches minces du dioxyde de titane dans les structures $\mathrm{TiO}_{2} / \mathrm{c}-\mathrm{Si}$ et $\mathrm{TiO}_{2} /$ verre, ont été déposées par pulvérisation cathodique radio fréquence réactive. Le plasma de pulvérisation est formé d'un mélange d'argon et d'oxygène. La pression totale étant maintenue à $10^{-2}$ mbar alors que la proportion d'oxygène dans le mélange varie de $5 \%$ à $20 \%$.

Pour déterminer les caractéristiques structurales, électroniques et optiques des couches minces du dioxydes de titane $\left(\mathrm{TiO}_{2}\right)$ nous avons utilisé un ensemble de techniques d'analyses complémentaires: la diffraction des rayons $\mathrm{X}$ en incidence rasante, la réflectométrie des rayons $\mathrm{X}$ et les spectroscopies de photoémission $\mathrm{X}$ et de transmission optique.

Les principaux résultats obtenus au cours de l'étude des films minces du dioxyde de titane préparés à $10^{-3}$ mbar d'oxygène peuvent se résumer comme suit:

- les couches de $\mathrm{TiO}_{2}$ sont plycristallines. Elles sont formées d'un mélange de deux phases du dioxyde de titane: la rutile et l'anatase.

- le titane est entièrement oxydé. La composition chimique Ti:O des films est 1:2.02.

- les couches minces du dioxyde de titane sont transparentes et non absorbantes dans le visible. Il ont un indice de réfraction extrapolé à l'infini (infra-rouge) de 2.23. l'indice de réfraction dans le visible est de 2.45. La détermination du coefficient d'absorption en fonction de l'énergie a permis de déterminer une valeur du gap égale à $3.35 \mathrm{eV}$.

\section{Références}

[1] Macleod, H. A. (1986). Thin Film Optical Filters. Adam Hilger Ltd, Bristol, p. 391.

[2] Williams, L. M. and Hess, D. W. (1983). Structural properties of titanium dioxide films deposited in an rf glow discharge. J. Vac. Sci. Technol., A1, 1810.

[3] Pawlewiez, W. T., Hays, D. D. and Martin, P. M. (1980). High-band-gap optical coatings for 0,25 and 1,06 micron fusion layers. Thin Solid Films, 73, 169.

[4] Yoldas, B. (1980). Investigations of porous oxide as antireflective coating for glass surfaces. Appl. Opt., 19, 1425.

[5] Gulsen, G. and Naci Inci, M. (2002). Thermal optical properties of $\mathrm{TiO}_{2}$ films. Optical Materials, $18,373$.

[6] Huang, N. K., Wang, D. Z., Lu, Z. and Lin, L. B. (1994). X-ray photoelectron spectroscopy characterization of $\mathrm{TiO}_{2}$ films deposited by dynamic ion beam mixing. Surface and Coatings Technology, 70, 69 .

[7] Wicaksana, D., Kobayashi, A. and Kinbara, A. (1992). Process effects on structural properties of $\mathrm{TiO}_{2}$ thin films by reactive sputtering. J. Vac. Sci. Technol. A, 10(4), 1479.

[8] Benett, J. M., Pelletier, E., Albrand, G., Borgogno, J. P., Lazarides, B., Carniglia, C. K., Schmell, R. A., Allen, T. H., Tuttle-Hart, T., Guenther, K. H. and Saxer, A. (1989). Comparison of the properties of titanium dioxides films prepared by various techniques. Appl. Opt., 28, 3303.

[9] Leprince-Wang, Y., Yu-Zhang, K., Nguyen Van, V., Souche, D. and Rivory, J. (1997). Correlation between microstructure and the optical properties of $\mathrm{TiO}_{2}$ thin films prepared on different substrates. Thin Solid Films, 30, 738 .

[10] Leinen, D., Espinos, J. P., Fernandez, A. and Gonzalez-Elipe, A. R. (1994). Ion beam induced chemical vapor deposition procedure for the preparation of oxide thin films: Preparation and characterization of $\mathrm{TiO}_{2}$ thin films. J. Vac. Sci. Technol. A., 12(5), 2728.

[11] Ijdiyaou, Y., Hafidi, K., Azizan, M., Ameziane, E. L., Outzourhit, A., Dreesen, L. and Benai, K. (1998). Grazing incidence X-ray reflectometry studies of $\mathrm{CdTe}(111)$ surfaces and a-Si thin films. Solar Energy Materials and Solar Cells, 52, 27.

[12] Ijdiyaou, Y., Hafidi, K., Azizan, M., Ameziane, E. L., Patrat, G., Brunel, M., Ortega, L. and Nguyen Tan, T. A. (1995). The formation of sputtered Ta/a-Si and a-Si/Ta interfaces in a-Si/Ta/a-Si/c-Si structure. Thin Solid Films, 266, 224.

[13] Schiller, S., Beister, G. and Sieber, W. (1981). The effect of substrate temperature and oxygen pressure on the structural properties of dc reactive plasmatron sputtered titanium oxide films. Thin Solid Films, 83, 239.

[14] Suhail, M. H., Mohan Rao, G. and Mohan, S. (1992). dc reactive magnetron sputtering of titanium-structural and optical characterization of $\mathrm{TiO}_{2}$ films. J. Appl. Phys., 71(3), 1421.

[15] Wiggins, M. D., Nelson, M. C. and Aita, C. R. (1996). Phase development in sputter deposited titanium dioxide. J. Vac Sci. Technol. A, 14(3), 772.

[16] Guinier, A. (1969). Theorie et Technique de Radiocristallographie. Dunod Editeur, Paris.

[17] Croce, P. and Nevot, L. (1976). Etude de couches minces et des surfaces par réflexion rasante spéculaire ou diffuse de rayons X. Rev. Phys. Appl., 11, 113.

[18] Handbook of Chemistry and Physics. Lide, D. R. (Ed.-in-Chief). 73rd ed (1992-1993). 
[19] Croce, P., Nevot, L. and Pardo, B. (1972). Contribution à l'étude des couches minces par réflexion spéculaire de rayons X. Nouv. Rev. d'Optique. Appliquée, 3(1), 37.

[20] Scofield, J. H. (1976). Hartree-Slater subshell photoionization cross-sections at 1254 and 1487 eV. J. Electron. Spectrosc. Rel. Phen., 8, 129.

[21] Lollman, D. B. B. (1992). Etude des propriétés électroniques et structurales du système $\mathrm{Er} / \mathrm{Si}(111)$. Thèse de Doctorat Grenoble I.

[22] Oh, W. S., Xu, C., Kim, D. Y. and Goodman, D. W. (1997). Preparation and characterization of epitaxial titanium oxide films on $\mathrm{Mo}(100)$. J. Vac. Sci. Technol. A, 15(3), 1710.

[23] Sanjinès, R., Tang, H., Berger, H., Gozzo, F., Margaritondo, G., and Lèvy, F. (1994). Electronic structure of anatase $\mathrm{TiO}_{2}$ oxide. J. Appl. Phys., 75(6), 2945.

[24] Atanassov, G. Thielsch, R. and Popov, D. (1993). Optical properties of $\mathrm{TiO}_{2}, \mathrm{Y}_{2} \mathrm{O}_{3}$ and $\mathrm{CeO}_{2}$ thin films deposited by electron beam evaporation. Thin Solid Films, 223(2), 288.

[25] Pulker, H. K. (1984). Coatings on Glass. Elsevier Science Publishers B. V., p. 311.

[26] Bennouna, A., Laaziz, Y. and Idrissi, M. A. (1992). The influence of thickness and index inomogeneities on the transmission of semiconductor thin films. Thin Solid Films, 213, 55.

[27] Bruhat, G. (1959). In: Kastler, A. (Ed.), Cours de Physique Générale (Optique). Masson et Cie, Paris p. 372.

[28] Laube, M., Rauch, F., Ottermann, C., Anderson, O. and Bange, K. (1996). Density of thin $\mathrm{TiO}_{2}$ films. Nuclear Instruments and Methods in Physics Research B, 113, 288.

[29] Narashimha, K. R. and Mohan, S. (1990). Optical properties of electron-beam evaporated TiO films deposited in an ionized oxygen medium. J. Vac. Sci. Technol. A, 8(4), 3260.

[30] Tang, H., Prasad, K., Sanjinès, R., Schmid, P. E. and Lévy, F. (1994). Electrical and optical properties of TiO anatase thin films. J. Appl. Phys., 75(4), 2042. 

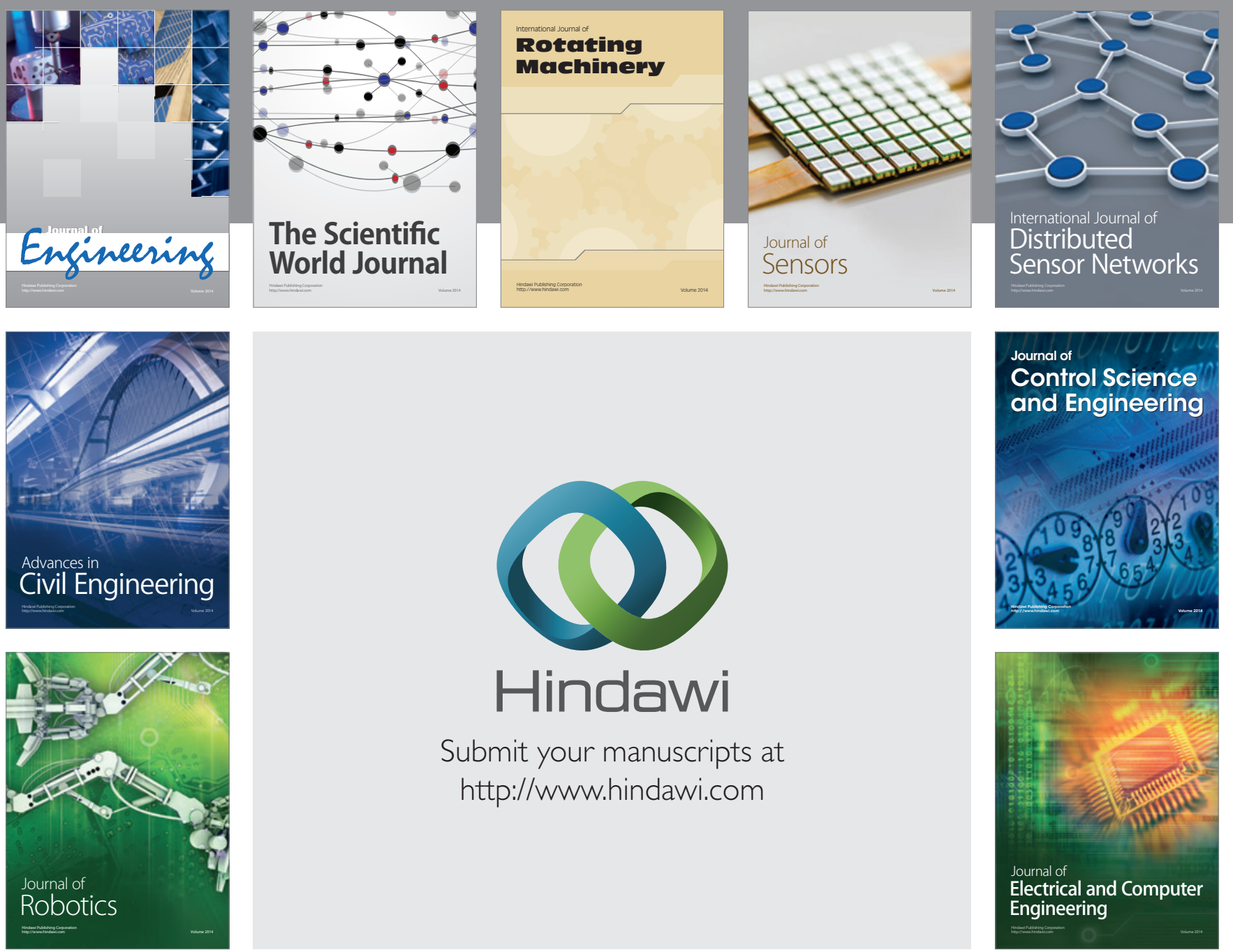

Submit your manuscripts at

http://www.hindawi.com
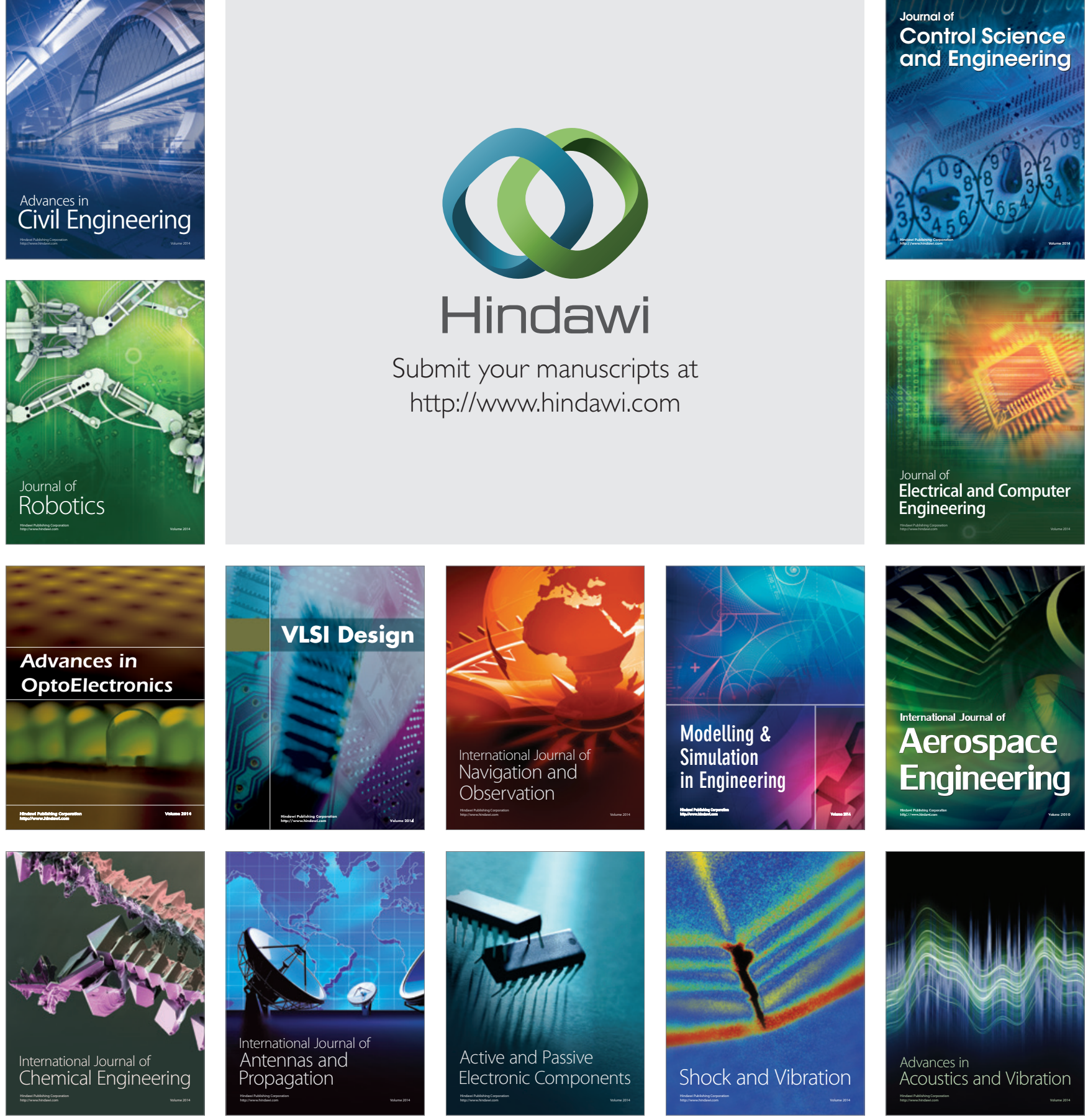